\title{
PROTON-PROTON FEMTOSCOPY AND ACCESS TO DYNAMICAL SOURCES AT INTERMEDIATE ENERGIES
}

\author{
E.V. Pagano ${ }^{1,2, a}$, G. Verde ${ }^{3}$, T. Minniti ${ }^{1}$, P. Danielewicz ${ }^{4}$, B. Barker ${ }^{4}$, and LASSA-Miniball collab- \\ oration \\ ${ }^{1}$ Universita' di Catania, Dipartimento di Fisica e astronomia, Catania, Italy \\ ${ }^{2}$ INFN-LNS, Via Santa Sofia 62, Catania, Italy \\ ${ }^{3}$ INFN-Sezione di Catania, Via Santa Sofia 64, Catania, Italy \\ ${ }^{4}$ Michigan State University-NSCL, East Lansing, Michigan, 48824
}

\begin{abstract}
Accessing dynamics in heavy-ion collisions represents a priority in nuclear physics, due to its links to the isospin dependence of the nuclear Equation of State and the space-time properties of systems under extreme conditions produced during the reaction. By means of particle-particle correlations it is possible to probe such space-time properties, allowing one to learn about the space-time properties of the source of particle emission. In this poster presentation we show an application of imaging techniques to study the two-proton source function at the dynamical stage of the reaction.
\end{abstract}

\section{Introduction}

Energetic heavy-ion collisions allow one to study the properties of nuclear matter under extreme conditions. In central collisions, after an early stage of pre-equilibrium emission and compression (strongly sensitive to the nuclear equation of state), the density drops to sub-saturation $\left(\rho<0.17 \mathrm{fm}^{-3}\right)$, undergoing fragmentation phenomena. Since the dynamics occur within this wide rage of densities, there can be sensitivity to the EoS. Moreover, access to N/Z-asymmetric beams permits investigations of the isospin dependence of the EoS. It is expected that these systems allow to produce nuclear systems over a range of densities and explore the isospin dependent equation of state [1,2]. The dynamical evolution of heavy-ion collision systems is very complex and occurs over very short time scales, thus requiring observables that are sensitive to space-time properties. In particular it is important to isolate dynamically emitting sources that are strongly affected by the density dependence of the symmetry energy $[2,3]$. Proton-proton correlation functions have extensively been used as spacetime probes of reaction dynamics $[4,5]$. The sensitivity of emitting source space-time profiles to the pre-equilibrium emission stage of the reaction is especially important because it promises to provide tools to access the density dependence of the symmetry energy [3]. In this short contribution we present a study of two-proton correlation functions in $\mathrm{Xe}+\mathrm{Au}$ at $\mathrm{E} / \mathrm{A}=50 \mathrm{MeV}$. The emitting source functions, extracted by imaging techniques, are studied. The simultaneous presence of long-lived and short-lived sources significantly affects the measured correlation functions. BUU model simulations

ae-mail: epagano@lns.infn.it 
are under study to find observables that help isolate the short-lived portion of the underlying emitting source which is dominated by emissions of particles in the early dynamical stage of the reaction $(\mathrm{t}<100 \mathrm{fm} / \mathrm{c})$. This preliminary study uses correlation observables and imaging techniques to improve comparisons between data and transport model simulations with the aim of exploring different transport properties of the studied systems, including effects of the symmetry energy [6-8].

\section{Experimental Setup and result}

The data were collected using the LASSA array, consisting of nine telescopes, each one having two layers of Double Sided Silicon Strip Detector (DSSSD) and four CsI crystals [9]. This high angular resolution array was coupled to the Miniball/Miniwall array [10] used to better isolate central collision events. This is accomplished by selecting a high multiplicity of charged particles detected by the combined array Miniball/Miniwall+LASSA, Mult $_{T o t}>25$. This selection, imposed also by the typical needs of correlation measurements requiring high statistics, does not exclude the presence of semicentral collisions where a forward moving projectile-like fragment (PLF) is produced. Fig.1 [13] shows proton kinetic energy spectra measured at three polar angles. These spectra contain different contributions: pre-equilibrium sources dominated by nucleon-nucleon collisions and characterizing the early stage of the reaction; emissions at mid-rapidity by the overlapping regions of projectile and target nuclei; evaporations by PLF sources and by secondary decays of excited primary fragments. These contributions are characterized by different emission time-scales that significantly affect the line-shape of the measured two-proton correlation function, $1+R(q)$, which is shown on the middle and right panels of Fig.2. The correlation function is defined as

$$
1+R(\mathbf{q})=C_{12} \cdot \frac{Y_{12}\left(\mathbf{p}_{1}, \mathbf{p}_{2}\right)}{Y_{m i x}\left(\mathbf{p}_{1}, \mathbf{p}_{2}\right)} .
$$

$Y_{12}\left(\mathbf{p}_{1}, \mathbf{p}_{2}\right)$ is the two-particle coincidence yield constructed with pairs detected in the same event, while $Y_{12}\left(\mathbf{p}_{1}, \mathbf{p}_{2}\right)$ is the uncorrelated two-particle yield constructed with pairs of protons chosen from different events (event mixing). $q$ is the momentum of relative motion in the two-proton system, $q=\frac{1}{2}\left|\vec{p}_{1}-\vec{p}_{2}\right|$ and $C_{12}$ is a normalization constant obtained by imposing that $R(\mathbf{q}) \approx 0$ for very large values of $q$. Short-lived (fast) sources dominate the shape of the large peak at $q \approx 20 \mathrm{MeV} / \mathrm{c}$, while long-lived evaporative and secondary decay sources contribute to significantly attenuate the amplitude of the peak [11-13]. A detailed study of the shape of the peak thus provides tools to constraints and explore these sources. This can be accomplished by solving the Koonin-Pratt (KP) equation [4]:

$$
1+R(\mathbf{q})=1+\int d \mathbf{r} S(\mathbf{r}) \cdot K(\mathbf{r}, \mathbf{q})
$$

where the source function, $S(\mathbf{r})$, is defined as the probability of emitting two protons at relative distance $\mathbf{r}$ calculated at the time when the second proton is emitted, and the $K(\mathbf{r}, \mathbf{q})$ is the known kernel function that incorporate the particle-particle scattering wave function (see Ref. [5] for details).

The Koonin-Pratt equation is commonly solved by using a Gaussian approach. In the case of angle-averaged correlation functions, the source function, $S(r)$, is assumed to be Gaussian-shaped, i.e. $S(r) \propto \lambda \cdot e^{-r^{2} / 2 r_{0}^{2}}$. The main parameter of this gaussian profile, i.e. the width or "size", $r_{0}$, is then extracted by fitting the KP equation to the experimental data [14]. In order to take into account possible contributions by multiple sources characterized by different emission time scales, the $\lambda$ parameter represents the fraction of proton pairs produced at the dynamical stage of the reaction by short lived emitting sources dominated by nucleon-nucleon collision processes. These approaches may be affected by model-dependencies due to a priori assumptions about the shape of the emitting source. 


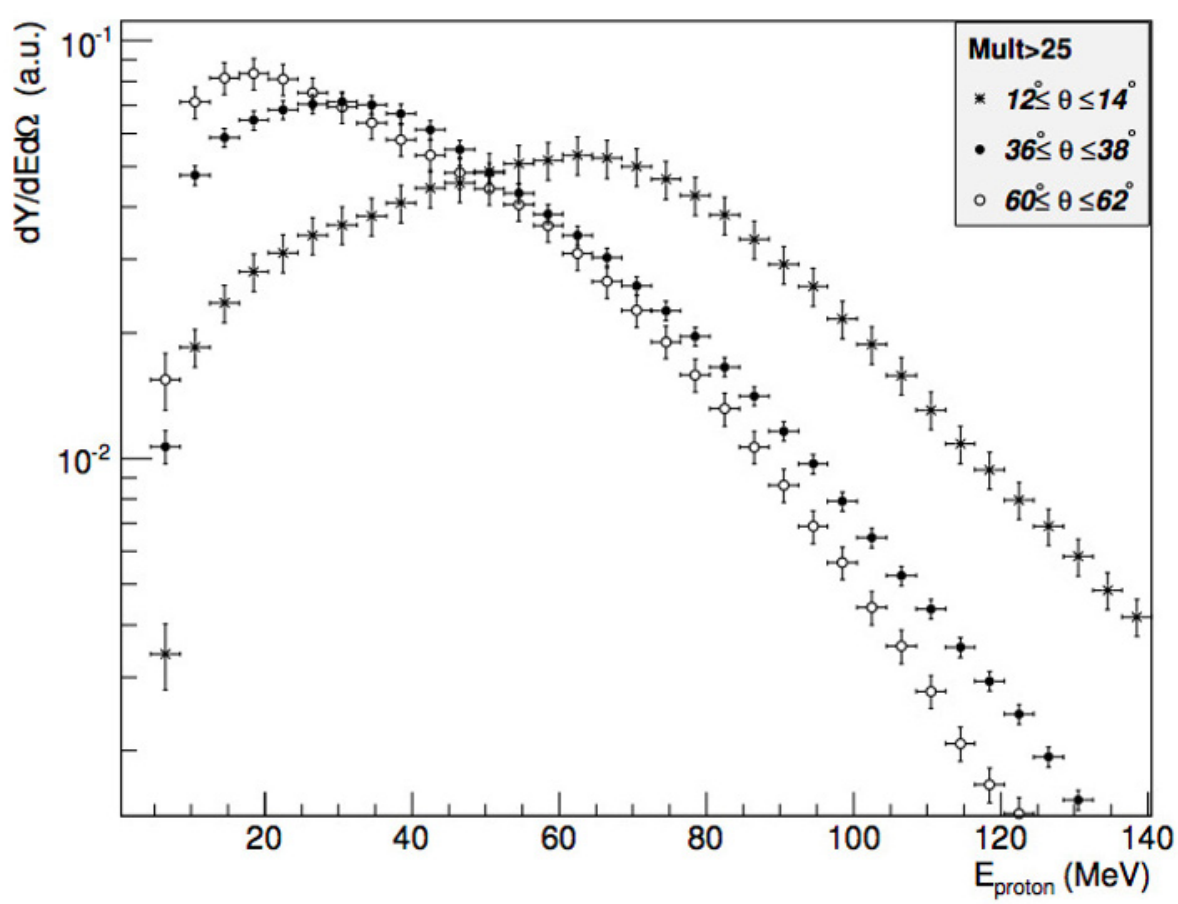

Figure 1. Proton energy spectra measured in semi-central collisions at polar angles of $13^{\circ}$ (crossed dots), $37^{\circ}$ (filled dots) and $61^{\circ}$ (open dots).

More recently, less model-dependent "imaging" approaches have been proposed in order to extract the profile of the source function by numerical inversion of the KP equation [11, 15]. In the imaging approach the source profile is extracted with reduced a priori parameterizations, allowing one to extract non-Gaussian shapes. Considerations about the physics content of these observables link the integral of the extracted profile, $\lambda_{\text {fast }}=4 \pi \int_{0}^{r_{\max }} S(r) r^{2} d r$, to the fraction of proton pairs emitted by the fast source. The integral of the source is evaluated up to an upper limit, $r_{\max }$, which represents the maximum r-value that can be imaged within the measured q-value range in the experimental correlation function (see [11] for details).

Having reduced model dependencies and the number of a priori assumptions on the shape of the two-particle source function, the profile extracted by imaging techniques represents a more realistic approach to the solution of the Koonin-Pratt equation. The "size" parameter then is simply deduced directly from the width of the imaged profile shown on the left panel of Fig. 2. As a quantitative measure of this width we choose the FWHM (full-width at half maximum of the S(r) profile), obtaining $r_{\frac{1}{2}}=4.4 \pm 0.5$. This source size parameter unequivocally linked to the width of correlation peak at $20 \mathrm{MeV} / \mathrm{c}$ which needs to measured with high accuracy $[12,13]$. Moreover, the integral of the extracted profile, $\lambda_{\text {fast }}=0.18$, represents the fraction of measured proton pairs attributed to the emission at the dynamical stage of the reaction. Then the fraction of protons emitted by the fast source is approximately given by $f=\sqrt{\lambda_{\text {fast }}}$, corresponding to about $45 \%$ of all emitted protons during the reaction. The extracted profile can also be compared to BUU model simulations that predict a sensitivity of two-proton correlation functions to the density dependence of the symmetry energy [3]. 


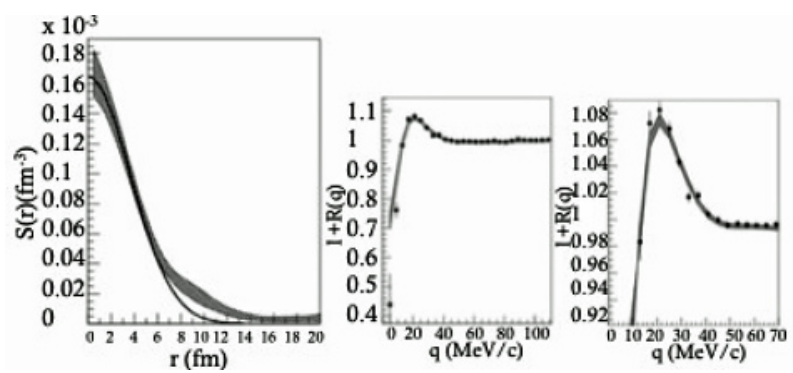

Figure 2. Two-proton correlation function (data points on the middle and right panels) measured in semi-central $\mathrm{Xe}+\mathrm{Au}$ collisions at $\mathrm{E} / \mathrm{A}=50 \mathrm{MeV}$ and results of the applied imaging techniques (thick bands). The extracted source functions extracted by imaging and Gaussian approaches are shown on the left panel, respectively, as a thick band and a solid line.

However, as it was pointed out in Ref. [6, 7], transport theory simulations often do not handle longlived decays properly. Such difficulties directly affect the integral of the two-proton source function. It will therefore be important to find observables that allow one to isolate, within BUU simulations, only particles emitted at the dynamical stage of the reaction and more properly use those particles to perform comparisons to experimental data. Preliminary results on these simulations show that high transverse momentum protons are preferentially emitted at the early dynamical stage $(t<100 \mathrm{fm} / \mathrm{c})$ [16]. By imaging high transverse momentum correlation functions one can therefore improve comparisons to transport models and search for isotopic effects on the shape of the correlation function. The developed ideas will indeed be used to study central ${ }^{112} \mathrm{Sn}+{ }^{112} \mathrm{Sn}$ and ${ }^{124} \mathrm{Sn}+{ }^{124} \mathrm{Sn}$ collisions at $\mathrm{E} / \mathrm{A}=50 \mathrm{MeV}$, in order to test the sensitivity of the imaged profiles to the density dependence of the symmetry energy or to other transport properties such as the density and isospin dependence of the nucleon-nucleon collision cross section.

\section{Conclusions and Outlooks}

In this short contribution a preliminary analysis of dynamical sources produced in $\mathrm{Xe}+\mathrm{Au}$ collisions at $\mathrm{E} / \mathrm{A}=50 \mathrm{MeV}$ is discussed. Proton-proton correlation functions are analyzed with an imaging approach to explore the space-time profile of their emitting source to be later used in comparisons to isospin-dependent transport model simulations [3]. The analysis is also aimed at improving future investigations with higher resolution arrays and with more efficient centrality selection criteria. Efforts are also currently in progress to determine the necessary method to compare model predictions to experimental data.

\section{References}

[1] M.B. Tsang et al., Phys. Rev. Lett. 102, 122701 (2009)

[2] Bao An Li et al., Phys. Rept. 464, 113 (2008)

[3] L.W. Chen et al., Phys. Rev. Lett. 90, 162701 (2003)

[4] S.E. Koonin, Phys. Lett. B 70, 43 (1977)

[5] G. Verde et al., Eur. Phys. J. A 30, 81 (2006)

[6] D.O. Handzy et al., Phys. Rev. C 94, 858 (1994). 
[7] G. Verde et al., Phys. Rev. C 67, 034606 (2003)

[8] R. Kotte et al., Eur. Phys. Jour. A23, 271 (2005)

[9] B. Davin et al., Nucl. Instr. and Meth. A 473, 302 (2001)

[10] R.T. De Souza et al., Nucl. Instr. and Meth. A 295, 109 (1990)

[11] G. Verde et al. Phys. Rev. C 65, 054609 (2002)

[12] E. V. Pagano Tesi di Laurea Magistrale in Fisica Nucleare, Universita' di Catania, A.A 20112012

[13] E.V.Pagano, Nuovo Cimento C 036, 04 (2013)

[14] W. G. Gong et al., Phys. Rev. C 43, 1184 (1991)

[15] D.A. Brown and P. Danielewicz, Phys. Rev. C 64, 014902 (2001)

[16] B. Barker NSCL-Michigan State University, PhD Thesis, 2013 
